\title{
High-frequency slowly drifting structures in solar flares
}

\author{
M. Karlický, F. Fárník, and H. Mészárosová \\ Astronomical Institute of the Academy of Sciences of the Czech Republic, 25165 Ondřejov, Czech Republic \\ e-mail: karlicky@asu.cas.cz, ffarnik@asu.cas.cz
}

Received 3 May 2002 / Accepted 27 August 2002

\begin{abstract}
Radio emission of four solar flares with high-frequency slowly drifting structures is presented. Three sub-classes of these structures were recognized. It is shown that the April 15, 2001 X14.4 flare started with the slowly drifting structure associated with a plasmoid ejection observed by TRACE in the $171 \AA$ line. The August 18, 1998 event presents an example of the drifting pulsation structure (DPS) which is well limited in frequency extent at both sides. A further example of the DPS, but followed by clouds of the narrowband dm-spikes, was observed during the November 23, 2001 flare. Finally, in the case of the April 12, 2001 flare, the drifting pulsation-continuum structure was recorded at the same time as the metric type II radio burst, i.e. in different frequency ranges. The slowly drifting structures were analyzed and in two cases their relation to hard X-ray emission was studied. Possible underlying physical processes are discussed assuming the plasmoid ejection model of eruptive solar flares.
\end{abstract}

Key words. Sun: activity - Sun: flares - Sun: radio radiation

\section{Introduction}

There are numerous types of radio emission of solar flares (McLean \& Labrum 1985; Isliker \& Benz 1994; Bastian et al. 1998; Jiřička et al. 2001). Their relationship to hard X-ray emission was studied in detail by Aschwanden et al. (1985, 1990). Recently a new type of radio emission - the slowly drifting pulsation structure (DPS) - was recognized (Karlický \& Odstrčil 1994; Karlický 1998; Hori 1999; Khan et al. 2002). It is usually observed at the very beginning of eruptive solar flares (Karlický et al. 2001). The radio observations of the October 5, 1992 flare revealed that the DPS was generated at times of plasmoid ejection (Ohyama \& Shibata 1998; Kliem et al. 2000). New positional measurements of the DPS by the Nancay Radioheliograph (Khan et al. 2002) confirmed this relationship.

Based on the MHD numerical simulations, Kliem et al. (2000) suggested that every individual burst in the DPS is generated by superthermal electrons, accelerated in the peak of the electric field in the quasi-periodic and burst regime of the magnetic field reconnection. On the other hand, the global slow negative frequency drift of the DPS was explained by a plasmoid propagation upwards in the solar corona towards lower plasma densities.

Recently, Hudson et al. (2001) identified a rapidly moving hard X-ray source, observed by the Yohkoh/HXT, associated

Send offprint requests to: M. Karlický, e-mail: karlicky@asu.cas.cz with the moving microwave source and the plasmoid ejection seen in the Yohkoh/SXT images. The association with the highfrequency slowly drifting burst was also reported. Furthermore, Klein et al. (2001) presented a radio spectrum of the July 14,2000 flare with the slowly drifting continuum at 10:2510:40 UT in the frequency range $2000-200 \mathrm{MHz}$. Therefore, it looks like not only the DPS but also the high-frequency drifting continua and other slowly drifting structures can be connected with the plasmoid ejection.

In this paper we present unique examples of slowly drifting structures with different fine structures. These examples can be thus considered as sub-classes of the high-frequency slowly drifting structures. At least two of them (April 15, 2001 and August 18, 1998) were connected with a plasmoid ejection. The April 12, 2001 event was associated with the metric type II burst and the November 23, 2001 event with the narrowband dm-spikes.

\section{Observations}

Among various events we selected four flares showing three sub-classes of high-frequency slowly drifting structures. These sub-classes are defined according to their appearances in the radio spectrum: a) slowly drifting structure (general case or that with complex fine structures), b) slowly drifting pulsation structure (DPS), and c) slowly drifting pulsation-continuum structure. Their examples follow. 


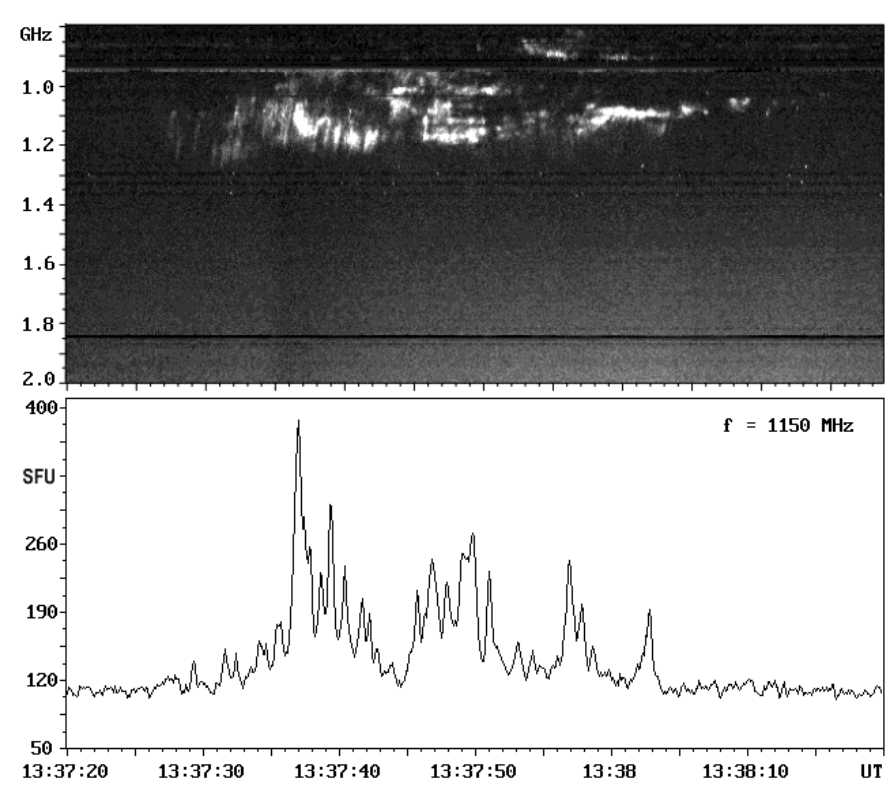

Fig. 1. The 0.8-2.0 GHz radio spectrum showing the slowly drifting structure at the very beginning of the April 15, 2001 flare (upper) and the radio flux plot at the frequency of $1150 \mathrm{MHz}$ (bottom) observed by the Ondřejov radiospectrograph.

\subsection{X14.4 flare with slowly drifting structure on April 15, 2001}

The flare belongs to the most intense flares in the present solar cycle. According to the GOES observations this flare started at 13:19 UT, its maximum was at 13:50 UT in soft X-rays, and it ended at about 15:30 UT; its importance reached X14.4. Simultaneously, the $\mathrm{H} \alpha$ flare of the importance 2B was reported in NOAA AR 9415 (at the position S20W85) at 13:36 UT, with maximum at 13:49 UT and ending at 15:35 UT. At the very beginning of this flare, in the $0.8-1.3 \mathrm{GHz}$ range a slowly drifting structure with the global negative frequency drift of $-4.7 \mathrm{MHz} \mathrm{s}^{-1}$ was observed between 13:37:27 and 13:38:10 UT (Fig. 1, where time profile at $1150 \mathrm{MHz}$ is also shown; see also Table 1). This slowly drifting structure had very interesting fine structures. It started with fast drift features resembling the high-frequency narrowband type III bursts in the 13:37:27-13:37:43 UT time interval. Their frequency drift was about $-270 \mathrm{MHz} \mathrm{s}^{-1}$. In the following periods these fast drifting features became narrower and narrower and they were replaced by several slowly drifting lines having a bandwidth of 30-50 MHz and a frequency drift in the interval of $-6-+4 \mathrm{MHz} \mathrm{s}^{-1}$. At the time of this slowly drifting structure, simultaneous TRACE observations (171 ̊ line, Fe IX, $0.9 \mathrm{MK}$ ) show plasmoid ejection (Fig. 2, upper part). The position of this plasmoid in comparison with the Yohkoh/SXT bright loops is shown in Fig. 2 (bottom part) by white contours. The speed of the plasmoid ejection in projection in the image plane was estimated to be $60 \mathrm{~km} \mathrm{~s}^{-1}$ in the upwards direction. In Fig. 3, a time comparison between the drifting structure and hard X-ray emission is shown. As one can see the drifting structure was observed simultaneously with the first weak increase of the hard X-ray emission of this very strong flare.
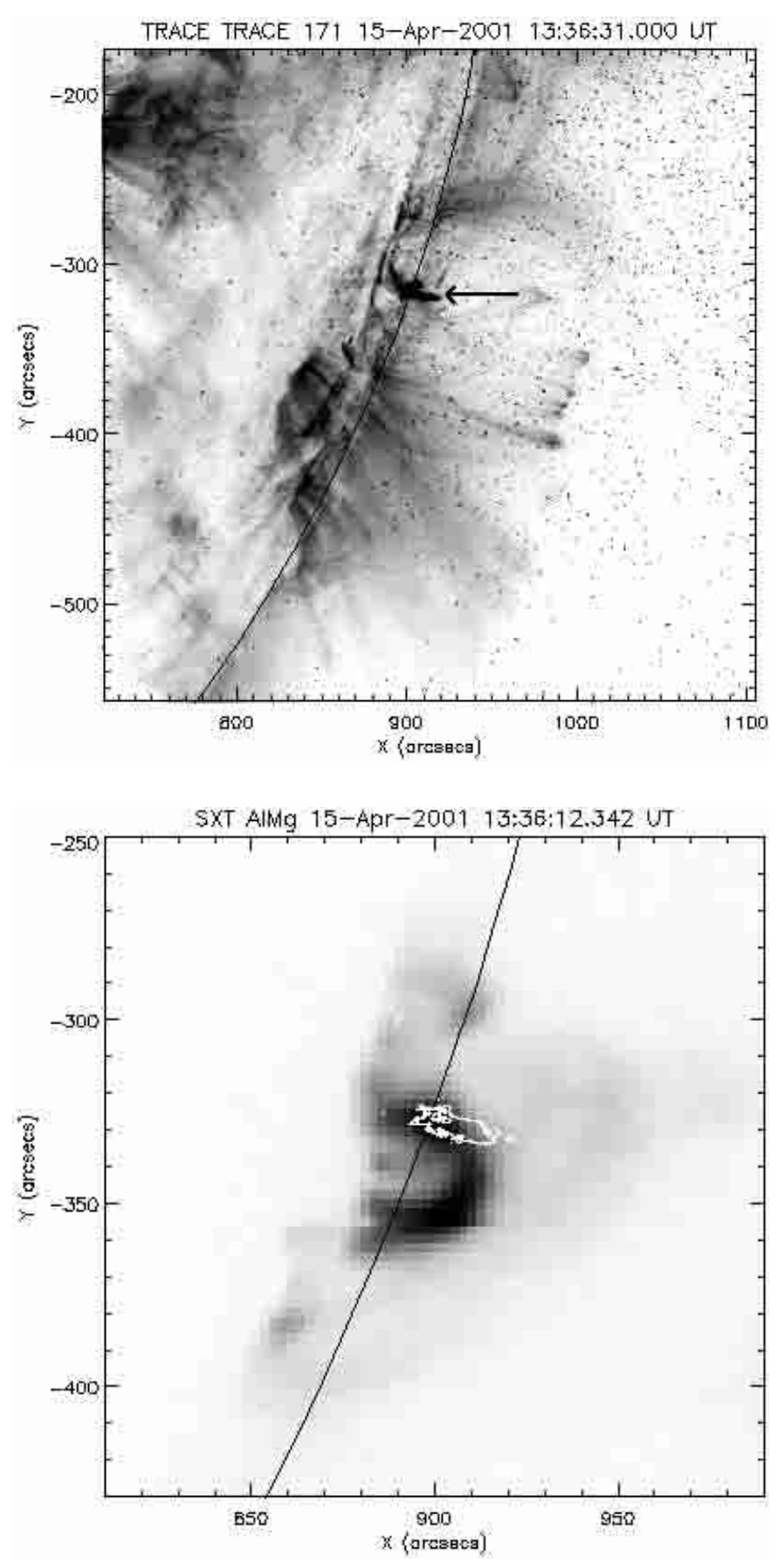

Fig. 2. Upper: TRACE $171 \AA \AA$ image of a plasmoid ejection (see the arrow) at the very beginning of the April 15, 2001 flare, at 13:36:41 UT, i.e. just before the drifting structure observation. Bottom: Yohkoh/SXT image at 13:36:12 UT; the white contours show the position of to the plasmoid observed by TRACE $171 \AA$ at 13:38:36 UT.

\subsection{Flare with slowly drifting pulsation structure on August 18, 1998}

The flare classified as $\mathrm{X} 2.8 / 1 \mathrm{~B}$ occurred at the east limb in NOAA AR8307 (N32E90) at 08:20 UT and was accompanied by an eruptive prominence, according to the Solar Geophysical Data event list. The hard X-ray emission as seen by the Hard X-ray Telescope (HXT) on board Yohkoh began as a gradual rise at $\sim 08: 18$ UT in all four energy bands of the instrument. 


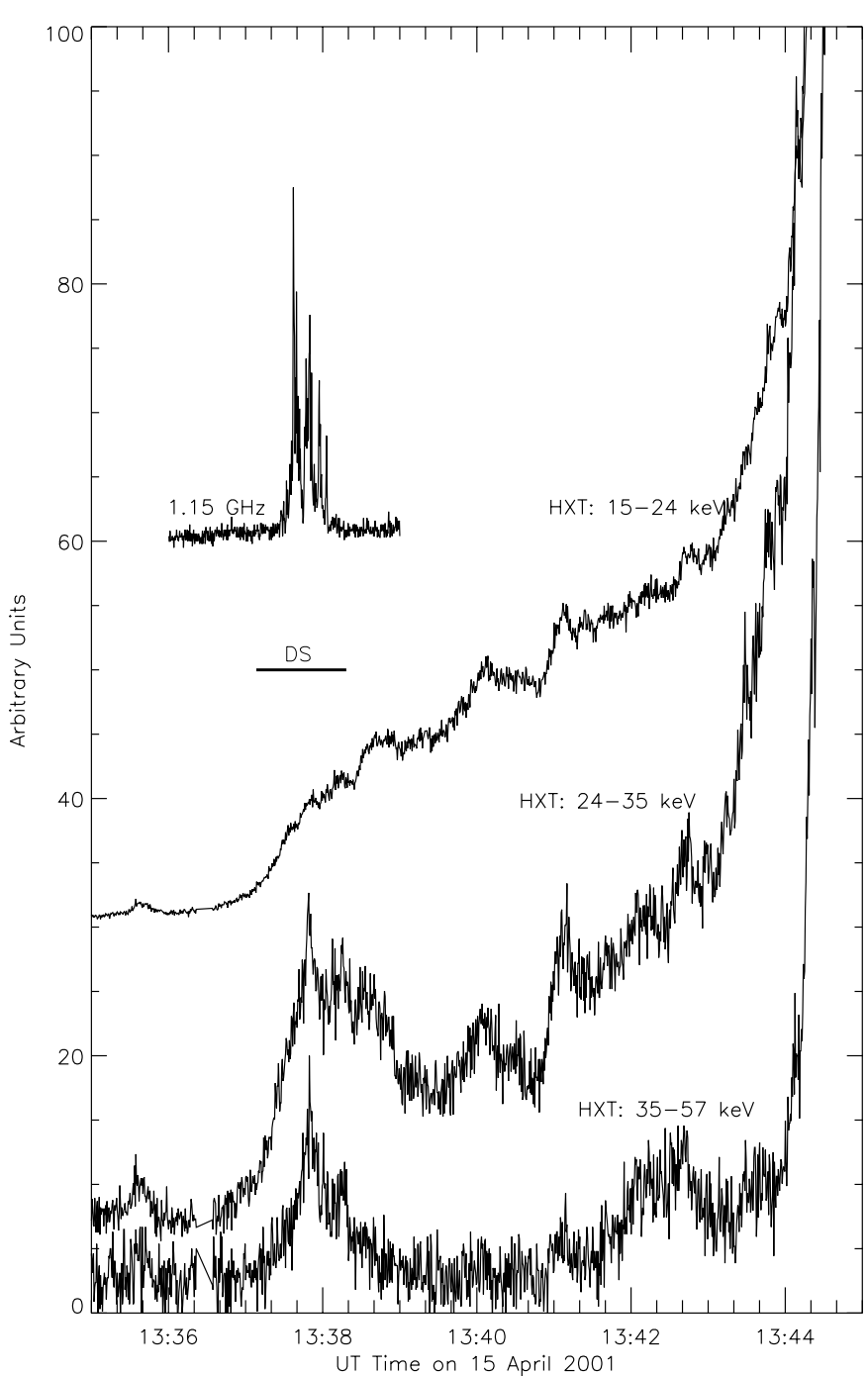

Fig. 3. Comparison of the radio flux at $1.15 \mathrm{GHz}$ (see also Fig. 1) with the hard X-ray emission in the 15-24 keV, 24-35 keV and 35$57 \mathrm{keV}$ energy bands observed by Yohkoh/HXT at the beginning of the April 15, 2001 flare. Interval of the drifting structure (DS) observation is depicted by the horizontal line.

An impulsive rise occurred at 08:19:30 UT in the M2 (33$53 \mathrm{keV})$ and $\mathrm{H}(53-93 \mathrm{keV})$ energy bands, followed by a series of pulses that lasted about $2 \mathrm{~min}$ in total. The HXT images show a loop-shaped source in the M2 band (33-53 keV) with an indication of a loop-top source component; this loop-top source is seen even in the H band image (53-93 keV). The Yohkoh Soft $\mathrm{X}$-ray Telescope (SXT) took an image series of the flare with different resolutions and filters that reveals an ascending motion of the blob structure (plasmoid) above the solar limb at times 08:19:54 to 08:21:32 UT. The plasmoid moved upwards with an average velocity (projected onto the image plain) of $\sim 490 \mathrm{~km} \mathrm{~s}^{-1}$ (for details see Karlický et al. 2002).

At radio waves, this flare started with a DPS at 08:18:30 UT, which stayed within the $0.8-2.0 \mathrm{GHz}$ band until 08:20:46 UT (Fig. 4; Table 1). This time interval nearly coincides with the rise of the hard X-rays from onset to peak and includes the formation and initial acceleration of the ascending emission blob in the soft X-ray images. Remarkably, this DPS

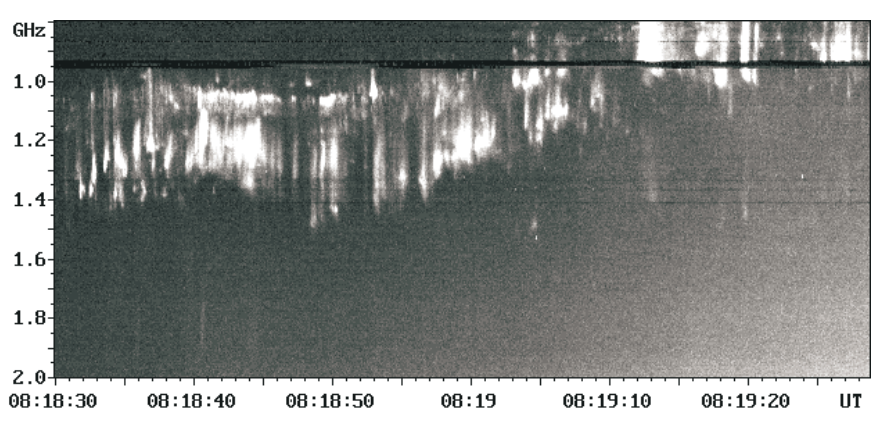

Fig. 4. The $0.8-2 \mathrm{GHz}$ radio spectrum observed by the Ondřejov radiospectrograph in August 18, 1998, showing the slowly drifting pulsation structure (DPS).

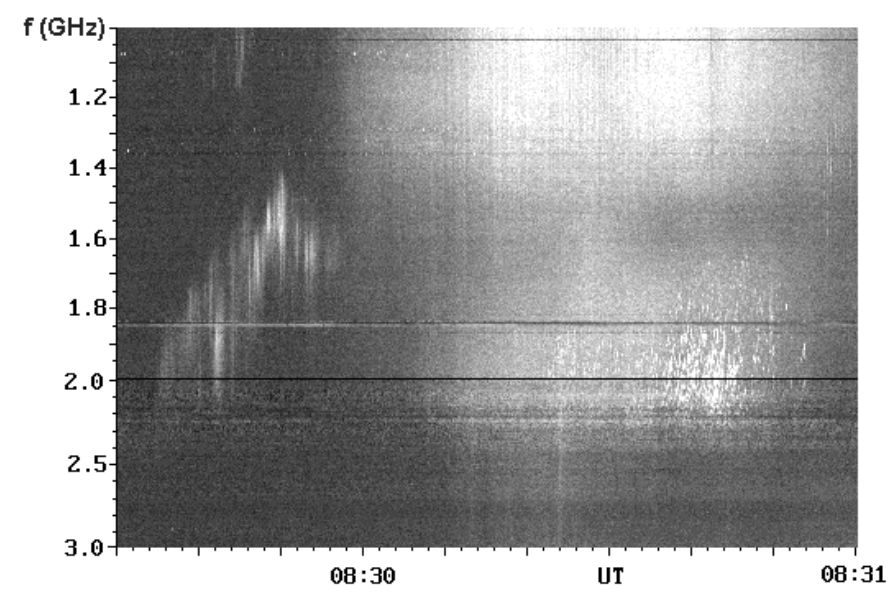

Fig. 5. Ondřejov radio spectrum in the $0.8-4.5 \mathrm{GHz}$ frequency range observed during the November 23, 2001 flare, showing an interesting sequence of the DPS between 08:29:35 and 08:30:00 UT, in the 1.4-2.2 GHz as well as clouds of the narrowband dm-spikes in the following period.

is well limited in frequency extent at both sides; most pulses are even amplified at the low and high-frequency edges, forming thus slowly drifting lines with a frequency drift in the interval of $-15-+3 \mathrm{MHz} \mathrm{s}^{-1}$. On the other hand, most of the individual pulses have infinite (not measurable) frequency drifts. However, among them there are several features with very high, but measurable frequency drifts (greater than $\pm 1 \mathrm{GHz} \mathrm{s}^{-1}$ ).

\subsection{Flare with slowly drifting pulsation structure and narrowband dm-spikes on November 23 , 2001}

Between 8:29 and 8:32 UT a very interesting radio spectrum with the DPS and narrowband dm-spikes was observed (Fig. 5, Table 1). The radio emission started with DPS between 08:29:35 and 08:30:00 in the 1400-2200 MHz range, which was followed by two clouds of narrowband dm-spikes between 08:30:00 and 08:31:30 UT in the frequency ranges $800-1500 \mathrm{MHz}$ and $1600-2500 \mathrm{MHz}$. The frequency drift of the DPS was $-30 \mathrm{MHz} \mathrm{s}^{-1}$. In lower frequencies, in the range of $40-80 \mathrm{MHz}$, these bursts were accompanied by a group of type III bursts (08:29:35-8:32:30 UT, Potsdam observations). All these radio emissions were produced by the C6.3 flare 


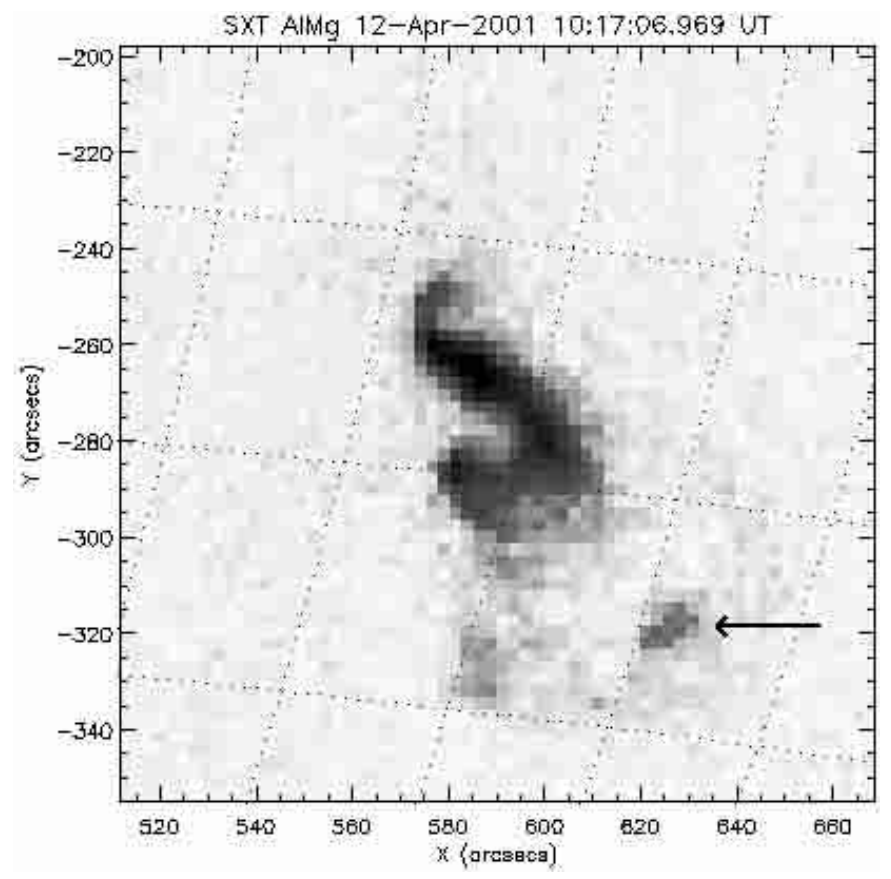

Fig. 6. The Yohkoh/SXT image of the April 12, 2001 flare at 10:17:07 UT. The arrow shows the bright blob.

observed by GOES at 08:28 UT with a maximum at 08:32 UT and ending at 08:36 UT. In the $\mathrm{H} \alpha$ line a flare of the importance SF was observed in the position S20W38 (in the NOAA AR 9704).

\subsection{Flare with slowly drifting pulsation-continuum structure and type II burst on April 12, 2001}

The X2.0 flare (according to the GOES classification) was observed in the NOAA AR 9415 at 09:39 UT, with its maximum at 10:28 UT, ending at 10:49 UT (NOAA Solar Events Report). The flare was located on the solar disc (Fig. 6); that is why recognition of the plasmoid (the bright and moving SXT blob at some coronal heights) is very difficult. We mention a bright feature (plasmoid?) which was observed at times after 10:16:30 UT (see the arrow in Fig. 6). The radio emission of this flare in the $40-4500 \mathrm{MHz}$ range is shown in Fig. 7. Here, not only type II bursts branches can be seen in the 40$700 \mathrm{MHz}$ range between 10:15 and 10:23 UT, but also the drifting pulsation-continuum structure in the frequency range of 450-1500 MHz between 10:17:20 and 10:22:00 UT. The drift rate of the DPS was $-1.6 \mathrm{MHz} \mathrm{s}^{-1}$. In this flare the slowly drifting structure was not the precursor of the metric type II burst; the slowly drifting structure was generated simultaneously with the type II radio burst, but in different frequency ranges. At the start time of the type II radio burst the position of the geometrical centrum of the radio source at $327 \mathrm{MHZ}$ at 10:17 UT was $E / W=0.73$ and $N / S=-0.27$ solar radii (Nancay Radio Heliograph observations), i.e. the position of $X=660, Y=240$ arcsecs in Fig. 6. The radius of this source (half of the maximum intensity) was about 70 arcsecs.

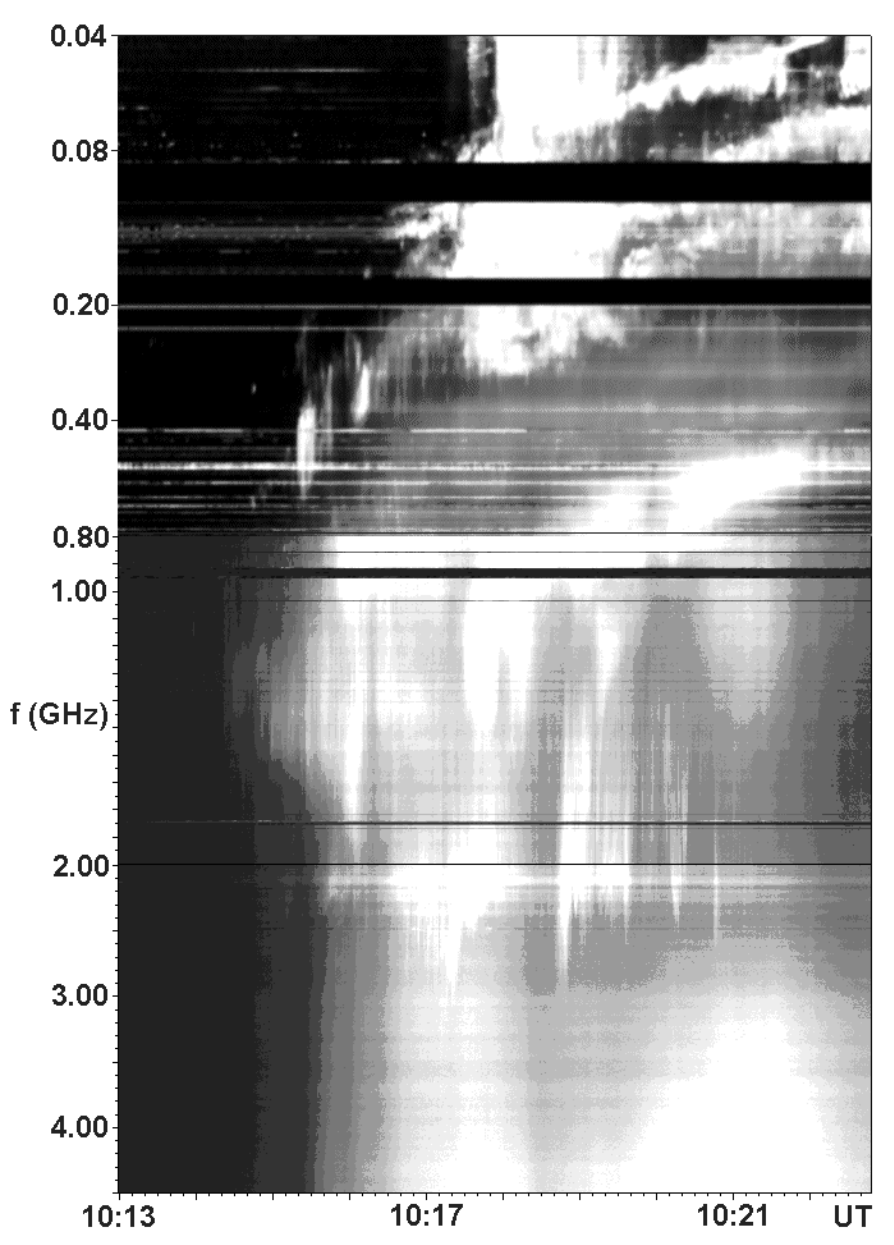

Fig. 7. Potsdam radio spectrum in the $40-800 \mathrm{MHz}$ band (courtesy Dr. A. Klassen) and Ondrejov radio spectrum in the $0.8-4.5 \mathrm{GHz}$ band observed during the April 12, 2001 event. The slowly drifting pulsation-continuum structure observed between 10:17:20 and 10:22:00 UT in the $0.45-1.5 \mathrm{GHz}$ range as well as the type II burst observed simultaneously in the metric frequency range are shown.

\section{Analysis of drifting structures}

Using the Fourier method the characteristic periods of the drifting structures at selected frequencies were determined. Results are summarized in Table 2, where the selected frequencies, periods and their statistical probabilities are shown. The results show a quasi-periodic character of these structures. To obtain more information about them, the histograms of pulse time separations at three selected cuts of the drifting structures were made and are shown in Fig. 8. As can be seen, the most frequent time separations are $0.8 \mathrm{~s}$ and $1.0 \mathrm{~s}$ in the drifting structure of August 18, 1998, $0.8 \mathrm{~s}$ and $1.4 \mathrm{~s}$ in the DS of April 15, 2001, and $0.4 \mathrm{~s}$ in the DS of November 23, 2001. On the other hand, in the case of the drifting pulsation-continuum structure of April 12, 2001 only the pulse time separations of 78, 26, and $10 \mathrm{~s}$ were determined.

Then, the relationship of the drifting structures with hard $\mathrm{X}$-ray emissions was studied by the cross-correlation method. For this purpose, only those drifting structures were selected which were observed at the very beginning of solar flares, where the radio emission is relatively simple. At the very 
Table 1. The basic characteristics of the presented slowly drifting structures.

\begin{tabular}{|c|c|c|c|c|}
\hline & Aug. 18, 1998 & Apr. 12, 2001 & Apr. 15, 2001 & Nov. 23, 2001 \\
\hline Start (UT) & $8: 18: 30$ & $10: 17: 20$ & $13: 37: 27$ & $8: 29: 35$ \\
\hline Duration (s) & 136 & 280 & 43 & 25 \\
\hline $\begin{array}{l}\text { Global freq. drift } \\
\left(\mathrm{MHz} \mathrm{s}^{-1}\right)\end{array}$ & -4.4 & -1.6 & -4.7 & -30 \\
\hline $\begin{array}{c}\text { Instantaneous } \\
\text { bandwidth (MHz) }\end{array}$ & 400 & $200-800$ & 300 & 300 \\
\hline $\begin{array}{l}\text { Drift of fine structures } \\
\qquad\left(\mathrm{MHz} \mathrm{s}^{-1}\right)\end{array}$ & $\begin{array}{l}\text { infinite, } \pm 1000 \\
\quad-15-+3\end{array}$ & infinite & $\begin{array}{c}-270 \\
-6-+4\end{array}$ & infinite \\
\hline Accompanied bursts & dm-continuum & $\begin{array}{c}\text { type II burst } \\
\text { dm-continuum }\end{array}$ & & $\begin{array}{l}\text { dm-spikes } \\
\text { m-type III }\end{array}$ \\
\hline Plasmoid ejection & yes & $?$ & yes & no information \\
\hline
\end{tabular}
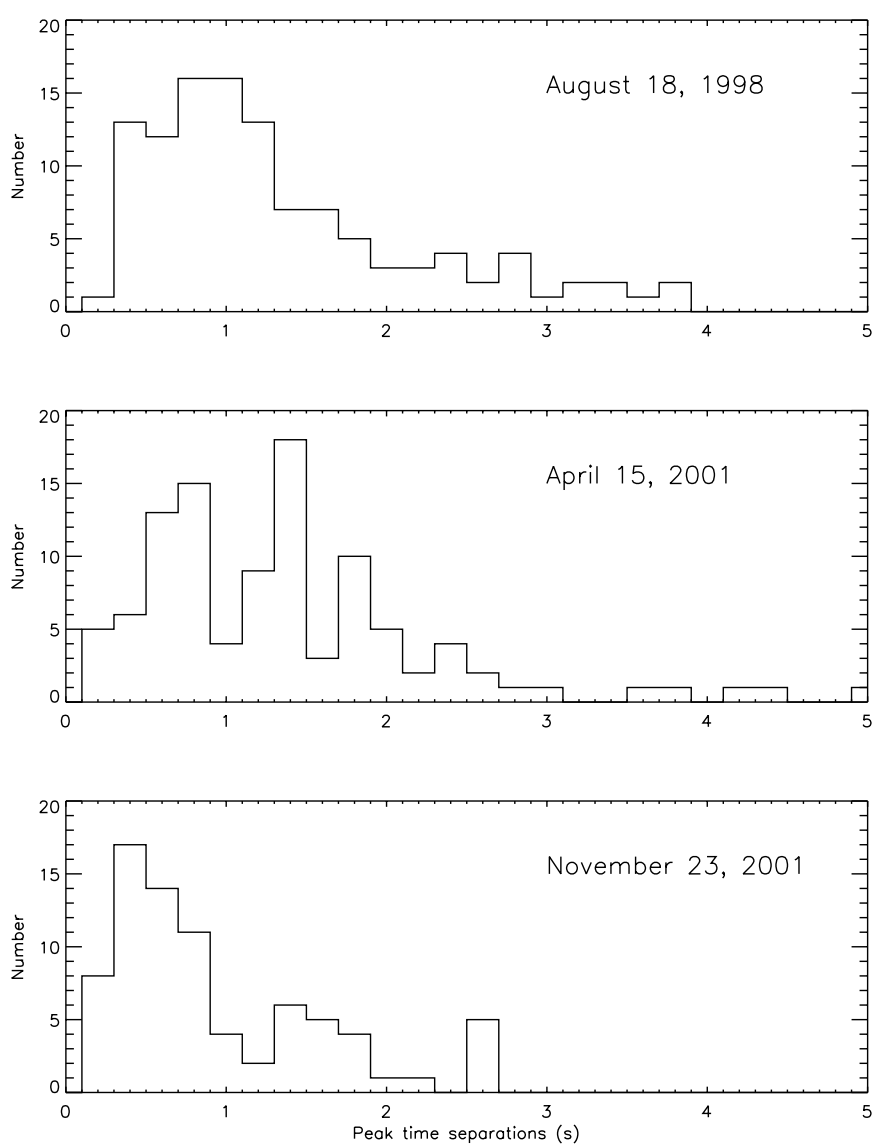

Fig. 8. Distributions of peak time separations in three drifting structures.

beginning of the November 23, 2001 flare the Yohkoh/HXT was not observing in the flare mode, therefore the comparison was made only for the April 15, 2001 and August 18, 1998 events. The drifting structures were integrated in frequencies and resulting time profiles were cross-correlated with the hard X-ray emission in the $35-57 \mathrm{keV}$ energy band. The cross-correlations are shown in Fig. 9. As seen in the case of the drifting structure of April 15, 2001 the correlation coefficient reached the value of 0.6 for the time lags of 5-15 s (thick line); the hard $\mathrm{X}$-rays are delayed after the radio emission. On the other hand, no significant correlation was found between hard X-rays and

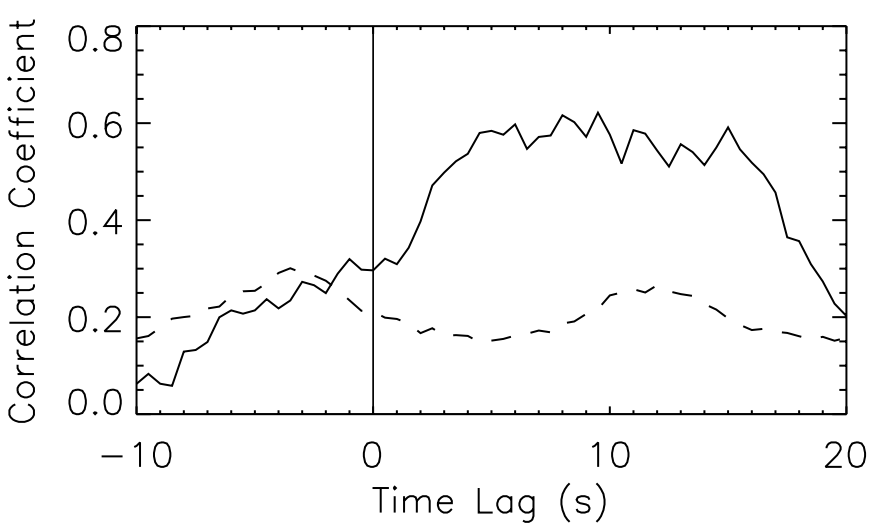

Fig. 9. Cross-correlation of the Yohkoh/HXT 35-57 keV X-ray flux and radio emission flux of the slowly drifting structures integrated over frequencies. Positive lag means a delay of hard X-ray emission after the radio. Thick line corresponds to the drifting structure in the April 15, 2001 event at 13:37:20-13:38:20 UT and the dashed line to that in August 18, 1998 at 8:18:30-8:19:30 UT.

drifting structure in the August 18, 1998 event (dashed line in Fig. 9, see also Table 2).

\section{Interpretation}

We assume that all sub-classes of the slowly drifting structures presented here have the same physical origin. Because at least two of them were associated with a plasmoid ejection, we interpret them using the model of solar flares with the plasmoid ejection (e.g. Shibata 1999; Yokoyama \& Shibata 2001). The plasmoid in such a model is considered to have a fully or partially closed magnetic field structure. In agreement with Kliem et al. (2000) we assume that in the burst regime of the magnetic field reconnection, in the current sheet below the ejected plasmoid the quasi-periodic electric field accelerates superthermal electrons which are injected into the plasmoid and then they generate separate pulses of drifting structures through a plasma emission mechanism. Thus, in the dependence on the magnetic field and density structures of the plasmoid, we can observe fast drifting features resembling type III bursts (at the beginning of the April 15, 2001 drifting structure and also some features in the August 18, 1998 event) or pulses with infinite frequency drifts (most of the features in the August 18, 1998 event and 
Table 2. Parameters of the presented slowly drifting structures. $T$ is the most frequent separation time (see histograms - Fig. 8).

\begin{tabular}{ccccc}
\hline \hline & Aug. 18, 1998 & Apr. 12, 2001 & Apr. 15, 2001 & Nov. 23, 2001 \\
\hline Selected frequency (MHz) & 1150 & 900 & 1150 & 1700 \\
Period (s) (probability) & $24.0(77.7 \%)$ & $75.0(87.0 \%)$ & $12.0(89.3 \%)$ & $3.0(91.7 \%)$ \\
& $8.6(72.5 \%)$ & $25.0(98.9 \%)$ & $2.5(80.7 \%)$ & \\
$T$ (s) & & $13.6(85.4 \%)$ & $1.5(79.1 \%)$ & \\
Cross-Correl. Coeff. & $0.8,1.0$ & $9.4(92.4 \%)$ & & 0.4 \\
Delay of hard X-rays (s) & 0.3 & & $0.8,1.4$ & \\
\hline
\end{tabular}

the November 23, 2001 event). The limited frequency extent of the drifting structures is naturally explained by a limited spatial extent of the plasmoid and the slow negative frequency drift by an expansion of this plasmoid and the corresponding plasma density decrease. On the other hand, when the injected flux of superthermal electrons into the plasmoid is nearly continuous, a drifting pulsation-continuum structure is observed (the April 12, 2001 case). An interesting example of the DPS associated with the narrowband dm-spikes was observed during the November 23, 2001 event. We expect such an association on the grounds of the model of the narrowband dm-spikes (Bárta \& Karlický 2001), which predicts the generation of spikes in the plasma reconnection outflows. In this event the DPS was very short and very early in the event, metric type III, hard $\mathrm{X}$-ray bursts, and the narrowband dm-spikes were observed. It looks like in this case the plasmoid was disrupted and superthermal electrons, generating the metric type III and hard $\mathrm{X}$-ray bursts, escaped. However at this stage of our knowledge, this is only a working hypothesis.

\section{Discussions and conclusions}

Four examples of the high-frequency slowly drifting structures were presented. According to their spectral characteristics we classified the drifting structures into three sub-classes. Their basic characteristics and parameters are summarized in Tables 1 and 2. A tendency of a decrease in time duration of the drifting structures with an increase in their global frequency drifts can be seen. At least two of the drifting structures were associated with the plasmoid ejection (August 18, 1998 and April 15, 2001), therefore the above-mentioned tendency can be connected with the stability of the plasmoid. The drifting pulsation-continuum structure of April 12, 2001 was accompanied by the type II burst (flare shock). This drifting structure is similar to those observed in the July 14, 2000 (Klein et al. 2001) and in the April 18, 2001 flares (Hudson et al. 2001). On the other hand, the remaining two drifting structures (August 18, 1998 and November 23, 2001) are similar to that observed in the October 5, 1992 flare (Kliem et al. 2000). Three of them (August 18, 1998, April 15, 2001 and November 23, 2001) were observed at the very beginning of the flares. For two events (August 18, 1998 and April 15, 2001) a relationship between the drifting structures and hard X-rays (35-57 keV) was studied by the cross-correlation method. In the August 18, 1998 event no significant correlation was found, and in the April 15, 2001 the relatively high correlation (the correlation coefficient equal to 0.6) was found between the radio emission of the DS and the hard X-rays shifted (delayed) by $5-15 \mathrm{~s}$ in the time axis. We think that these results of the cross-correlation can be explained by full (August 18, 1998) or partial (April 15, 2001) magnetic field isolation of the plasmoid. This idea agrees with the well-limited frequency extent of the DPS in the August 18, 1998 flare. On the other hand, in the case of the April 15, 2001 it takes 5-15 s for escape of the superthermal electrons, generating the DPS, out of the plasmoid trap and their penetration into dense atmospheric layers, where they produce hard X-rays.

Up to now, there are only three cases when crosscorrelations between DSs and hard X-ray emission were determined: the two present cases and a third one which was described in the paper by Kliem et al. (2000), where the anticorrelation (correlation coefficient of -0.4) was found. We think that this complex relationship is due to not only the magnetic field isolation of the plasmoid but, probably also, is due to the existence of two hard X-ray sources in the October 5, 1992 event: one source at the plasmoid as in the April 18, 2001 flare (Hudson et al. 2001) and a second one at the footpoints of the flare loops.

Although three types of slowly drifting structures (drifting structure with fast drifting features, drifting pulsation structure and drifting pulsation-continuum structure) are presented here, we interpret them in the same way. In agreement with Kliem et al. (2000) we assume that in the burst regime of the magnetic field reconnection in the current sheet below the ejected plasmoid, the quasi-periodic electric field accelerates the superthermal electrons which are injected into the plasmoid. Then these injected electrons generate separate drifting structures through a plasma emission mechanism. The difference between the subclasses of the drifting structures is explained by different density gradients and magnetic field structures of the plasmoid and, also, by a different injection process (quasi-periodic or continuous). The difference can also be given by different speeds of injected electron beams. Considering this model we can expect that some slowly drifting groups of narrowband dm-type III bursts also belong to the same class of radio bursts (i.e. the radio burst in Fig. 1a in the paper by Aschwanden et al. 1985).

Accepting these ideas and using the DS radio frequencies (450-1500 MHz), the electron plasma density in the plasmoid is in the range of $2.50 \times 10^{9}-2.77 \times 10^{10} \mathrm{~cm}^{-3}$ for the fundamental and $6.25 \times 10^{8}-6.94 \times 10^{9} \mathrm{~cm}^{-3}$ for the harmonic plasma emissions, respectively. These values are of the same order as those estimated by Ohyama \& Shibata (1998) and Hudson et al. (2001) for the October 5, 1992 and April 18, 2001 plasmoids, respectively. The finite frequency drifts in the April 15, 2001 
DS indicate density gradients in the plasmoid along the magnetic field lines. The time delay of the hard X-rays after the DS $(5-15 \mathrm{~s})$ in this case roughly corresponds to the collisional deflection time of superthermal electrons $\left(t_{\mathrm{d}} \approx 3.1 \times 10^{-20} v^{3} / n_{\mathrm{e}}\right.$, where $v$ is the electron velocity, Benz 1993), assuming their velocity as $c / 3$ ( $c$ is the speed of light) and the electron density in the plasmoid as $(2.0-4.5) \times 10^{9} \mathrm{~cm}^{-3}$ (harmonic radiation).

Very interesting observations were made during the April 12, 2001 flare where both the drifting structure and metric type II burst were observed simultaneously but in different frequency ranges. It indicates that this drifting structure was not a part of a large-scale travelling coronal shock front (type II radio burst).

Acknowledgements. The authors thank Dr. A. Klassen for providing the Potsdam-Tremsdorf radio spectrum and Dr. B. Kliem for discussions. This work was supported by the grants IAA3003202 of the Academy of Sciences of the Czech Republic and GAČR GA205/02/0980 under the key project K2043105 of the Astronomical Institute.

\section{References}

Aschwanden, M. J., Wiehl, H. J., Benz, A. O., \& Kane, S. R. 1985, Sol. Phys., 97, 159

Aschwanden, M. J., Benz, A. O., \& Kane, S. R. 1990, A\&A, 229, 206
Bárta, M., \& Karlický, M. 2001, A\&A, 379, 1045

Bastian, T., Benz, A. O., \& Gary, D. E. 1998, ARA\&A, 36, 131

Benz, A. O. 1993, Plasma Astrophysics (Kluwer Acad. Publ., Dordrecht, The Netherlands), 47

Hori, K. 1999, in Solar Physics with Radio Observations, Proceedings of the Nobeyama Symposium, NRO Report No. 479, 267

Hudson, H. S., Kosugi, T., Nitta, N., \& Shimojo, M. 2001, ApJ, 561, L211

Isliker, H., \& Benz, A. O. 1994, A\&AS, 104, 145

Jiřička, K., Karlický, M., Mészárosová, H., \& Snížek, V. 2001, A\&A, 375,243

Karlický, M. 1998, A\&A, 338, 1084

Karlický, M., \& Odstrčil, D. 1994, Sol. Phys., 155, 171

Karlický, M., Yan, Y., Fu, Q., et al. 2001, A\&A, 369, 1104

Karlický, M., Kliem, B., Mészárosová, H., \& Jiřička, K. 2002, Proc. of SPM10, Prague, in press

Khan, J. I., Vilmer, N., Saint-Hilaire, P., \& Benz, A. O. 2002, A\&A, 388,363

Klein, K.-L., Trottet, G., Lantos, P., \& Delaboudiniere, J.-P. 2001, A\&A, 373, 1073

Kliem, B., Karlický, M., \& Benz, A. O. 2000, A\&A, 360, 715

McLean, D. J., \& Labrum, N. R. (eds.) 1985, Solar Radiophysics (Cambridge, Cambridge University Press)

Ohyama, M., \& Shibata, K. 1998, ApJ, 499, 934

Shibata, K. 1999, Ap\&SS, 264, 129

Yokoyama, T., \& Shibata, K. 2001, ApJ, 549, 1160 